\title{
Employment of baby sign language in interactive learning for hearing-impaired people in Malaysia
}

\author{
N. T. J. Ong ${ }^{1}$, Ali S. H. A ${ }^{2}$, Yusoff A. H. $M^{3}$ \\ ${ }^{1,2}$ Faculty of Electrical and Electronic Engineering (FKEE), Universiti Tun Hussein Onn Malaysia (UTHM) \\ ${ }^{3}$ Faculty of Mechanical and Manufacturing Engineering (FKMP), Universiti Tun Hussein Onn Malaysia (UTHM)
}

\begin{tabular}{l} 
Article Info \\
\hline Article history: \\
Received Aug 20, 2019 \\
Revised Oct 21, 2019 \\
Accepted Nov 15, 2019 \\
\hline
\end{tabular}

Keywords:

Baby sign language

Hand gesture recognition

Interactive learning

Profound hearing impairment

\begin{abstract}
Communication is very important for people including adults and babies to exchange their thoughts, views and information. They can express their thoughts verbally and non-verbally. Baby sign language is non-verbal type that consists of a set of standard and organised form of hand gestures motion to represent different word. This language allows the children who have hearing impairment problem to communicate with others. In addition, it can also be taught to normal babies to convey their need before they have the ability to speak. It is very challenging for adults to understand babies and children with hearing impaired problem. Thus, a real-time hand gesture recognition system has been developed for adult to interactively learn the baby sign language. Firstly, the face region was detected in order to use the skin colour model of the face to detect the hand. After that, the hand shape was determined by finding the palm centre and convex hull. Convexity defects which representing gaps between the fingers were used to determine the number of fingertips whereas the movement of the hand was detected based on motion analysis. By setting the background colour to blue and adding extra light in front of the laptop, the system successfully obtained an accuracy higher than $85 \%$ rate for sign "mom", "eat" and "milk" when tested on Malay, Chinese and Indian races in Malaysia.
\end{abstract}

Copyright (ङ 2020 Institute of Advanced Engineering and Science. All rights reserved.

\section{Corresponding Author:}

Siti Hajar Aminah Binti Ali,

Department of Electronic Engineering,

Faculty of Electrical and Electronic Engineering (FKEE),

Universiti Tun Hussein Onn Malaysia (UTHM).

Email: aminahh@uthm.edu.my

\section{INTRODUCTION}

World Health Organization (WHO) has classified the hearing impairment into 5 categories which are type 0 or no impairment (with $\leq 25 \mathrm{~dB}$ mean hearing loss), type 1 or slight impairment (with 26-40 dB mean hearing loss), type 2 or moderate impairment (with 41-60 dB mean hearing loss), type 3 or severe impairment (with 60-80 dB mean hearing loss) and type 4 or profound impairment (with $\geq 81 \mathrm{~dB}$ mean hearing loss) [1]. Type 1 and type 2 are treatable using surgery, while type 2 and type 3 require the help of hearing aids to enable hearing process to occur. However, both surgical and hearing aids are unable to overcome type 4 hearing impairment. To enable communication for people with type 4 hearing loss to occur, cochlear implant, lip-reading and employment of sign language can be used [1]. Statistics from [2] estimated 16 millions (12-26 millions) children had hearing impairment excluding type 0 ( $\geq 35 \mathrm{~dB})$ in 2011 and it is expected to increase each year. The cost for hearing impairment treatment for low-income countries is high [3], therefore sign language is usually become the main chosen solution for communication for those who are having hearing loss.

Children with hearing impairment problem usually have the communication difficulty with other people. They are more likely to have negative behaviours as they become frustrated when their message is 
not understood by other people causing them to be hot-tempered and possess rage at all time [4-7]. These negative behaviours are usually occurred when is the children are trying to express their basic needs such as hunger, thirst or drowsiness to their parents. If they fail to get what they want, they will become agitated and cry all the time. Normally parents will send these children to attend a formal sign language classes or hire a professional babysitter to look after their children. However, for those who are from low-income family, they cannot afford to pay high salary of the babysitter and high course fee. As the consequence, these low-income parents will choose neither of these two instead they neglect their children causing them to lose the opportunities for learning.

It is important for all of us to learn baby sign language as baby sign language is the powerful mean of communication among children, not only for babies with profound hearing impairment problem but also for normal babies. For normal infants, learning baby sign language will help the infants below two years old to express their needs [8-9] before they can learn to speak properly. In addition, research from [10] has stated that about $20 \%$ of babies with hearing problem have parents who can hear normally. Thus, there is a small probability for everyone to have a child with hearing impairment. Due to its importance, this research focusses on learning baby sign language among adults, so that it can be used to teach the infants.

Nowadays, there are many kinds of medium of interactive learning such as the Internet and mobile apps which can help public people to learn the baby sign language by themselves without attending any baby sign language learning course. One of the sign language translation system has been developed for Arabic sign language which can be accessed on mobile devices which translating text into sign language animation [11-12]. In addition to this, there is also a development of sign language translation for learning English sign language [13] and learning Indian sign language [14]. Handheld mobile system has also been created for learning Arabic sign language where users can refer graphic applications to learn sign language [15]. There was a research done to develop sign language animation on mobile devices for learning Chinese sign language by providing 3-D virtual human model to teach the sign language [16]. However, there are still some limitations on the system due to the development of the translation system that is specific for certain country's language.

With the help of hand gestures to learn baby sign language, children especially those with profound hearing impairment problem can communicate with other people more naturally and expressively. This is because the hand gesture from them will be detected and translated automatically. It takes a great effort for children with profound hearing impairment to make a word and sentence to communicate to their parents. The recognition of finger detection for alphabets for Spanish sign language using contour analysis-based computer vision with input glove on the hand has found to be suitable for most letters as it can reach $99 \%$ of accuracy for some of the letters [17]. Boundary-trace based finger detection technique for alphabets of the American Sign Language (ASL) without any input of such special markers or gloves on the hand has found that it works accurately for $95 \%$ of the letters tested in the research work [18]. However, these techniques can only translate the alphabets.

Since learning hand gesture for baby sign language is good to understand infants especially for those who require special need, a real-time dynamic hand gestures recognition system is developed. Dynamic hand gesture recognition by using contour analysis to recognise Arabic language has $85.67 \%$ recognition rate where the testing has been done by different person with the same intensity of light [19]. A dynamic hand gesture recognition by using image processing module which developed in OpenCV has found that 9 of 11 hand gestures has worked properly with an efficiency of $70 \%$ with the same background colour [20]. The gesture recognition system by using Support Vector Machine (SVM) has found that the developed system is able to recognise the sign language accurately and it has to be in white background [21]. A few real-time recognition systems have been developed but the suitable background colour has not yet been studied.

The major objectives of this research is to develop an interactive learning of baby sign language that can be learnt effectively using Microsoft Foundation Class (MFC) Application and to investigate the performance of dynamic hand gesture recognition system in terms of accuracy among three main races in Malaysia. This project is primarily concerned on the motion hand gesture detection. The scopes of this study are about three basics baby sign language used such as "mom", "eat" and "milk". In this research, a built-in web-camera of a laptop was used to capture the image frame of the sign language. Two pre-setting experiments have been carried out which were testing on different light condition and different background color to gain the highest accuracy rate.

The rest of the paper is organised as follows. The proposed architecture for image processing is discussed in detail in Section 2. In section 3, the results of the proposed system are discussed. Section 4 concludes the paper. 


\section{RESEARCH METHOD}

A system of real-time dynamic hand gestures recognition is contributed by four major parts which are; (1) a regular web-camera, (2) Microsoft Visual Studio 2013, (3) OpenCV 2.4 .11 and (4) MFC Application. Figure 1 shows the proposed system architecture of the dynamic hand gestures. Firstly, an image frame is captured from the laptop web-camera. Next, the image frame is passed to the image processing module consists of the image filtering, image pre-processing and convex hull and convexity defects computation before being classified by using Microsoft Visual Studio 2013 and OpenCV. Finally, MFC Library is used to generate the applications in stable release state without running the program codes repeatedly. In this project, MFC application is used to visualise the real-time dynamic hand gestures and translate the gestures into appropriate meaning. The detail of image processing module is discussed in the following subsections.

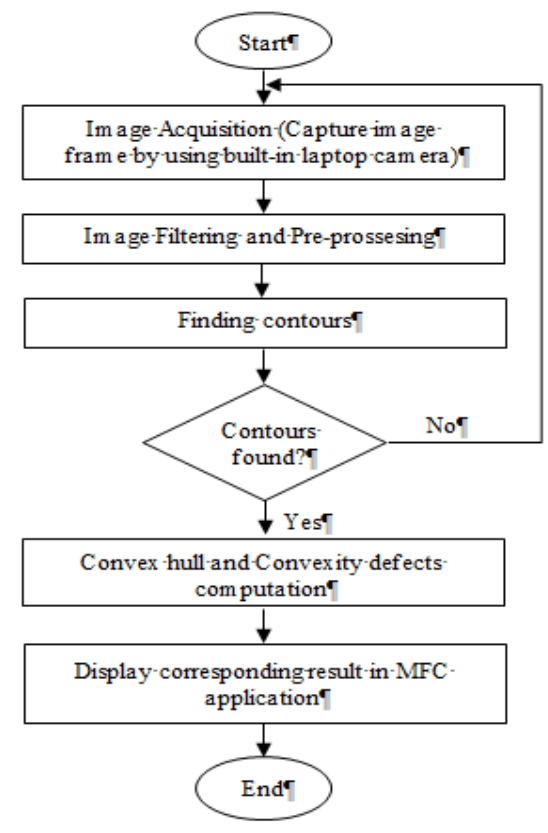

Figure 1. The flowchart of the dynamic hand gesture recognition

\subsection{Image Acquisition and Skin Colour Modelling}

Image Acquisition stage is the first stage of any vision system. In this stage, the image is retrieved from hardware-based source then passed through the other stage. The detected face colour is used to build the skin colour model. Skin colour model is more reliable as it is suitable for people with different skin colours and in different lightning conditions. The major factor that influences the image acquisition is the initial setup of the hardware that is used to capture the images. If the initial setup of the hardware is in low quality, the captured image may not be salvaged properly. Improper configuration and alignment of the software will then produce visual artifacts which can complicate the image processing [22].

\subsection{Image Filtering and Pre-Processing}

Image processing is a method to perform some operations on an image to obtain an enhanced image or extract some useful information from it. In this process, the input is the image whereas the output is the characteristics or features associated with the image. Image processing basically consists of three steps which are; importing the image via image acquisition tools, analysing and manipulating the image and producing an altered image or report which is based on the analysed image. The main process in this step is to only detect the hand region by applying skin colour detection. Thus, the face region which is detected before will be removed to avoid the system to be confused.

\subsection{Contours Convexity Hull and Convexity Defects Computation}

The contour which is linked to the next logical step is steeply encoded about the location of the next point on the curve to comprehend the shape of the processed image [23-24]. Figure 2 shows the image of 
hand with convex hull and convexity defects. Red line represents the convex hull of hand whereas the double-sided arrow represents the convexity defects [25]. The number of fingers is used to select specific actuator in the manipulator.

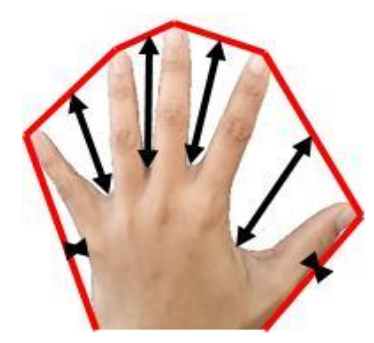

Figure 2. Image of hand with convex hull (red line) and convexity defects (doubled-sided arrow) [24]

Hand shape can be determined by finding the largest contour of the hand. Firstly, the palm centre is detected to find the maximum area of the palm of the hand. A blue circle will be formed to show the founded palm region whereas a white spot represents the detected palm centre. Then, the convex hull and convexity defects representing the gaps between fingers are detected to determine the number of fingers. The red spot resembles the detected convex hull whereas the light blue spots represent the detected gaps between the fingers.

The segmentation of hand motion can be done via motion analysis. Motion analysis enables any movement in the video scene to be detected. Hence, a uniform and static background should be set up to avoid the system from confusion and remove the noise. In this project, the centre of the hand which was detected in each frame has allowed its position to calculate the centre in each frame by calculating the tangent of the angle between the horizontal line and the line joining at the centre in consecutive frames. This enabled the system to identify the direction of the motion of the hand. Motion analysis then extracted the feature to obtain the temporal information and made decision based on the detected hand gestures.

The function mask is commonly used to copy the desired information of the hand gesture on the location of the convex hull, convexity defects, palm center, number of fingertips and the direction of hand movement. This region is usually set as the desired region whereas the other region will not be copied. The recorded information is set as the reference of the hand gesture and computed with the other hand gesture during the testing phase. Figure 3 shows the detected face image and the location of the convex hull for sign "mom", "eat" and "milk". The system recognises the hand gesture as "mom" when it detects five fingertips, four convexity defects and the distance becomes closer to the face region. For "eat" recognition, the detected fingertips should be more than three, the detected convexity defects should be less than three, and the distance between hand and face should be less than $5 \mathrm{~cm}$. Whereas for the "milk" recognition, the system has to detect zero fingertips.

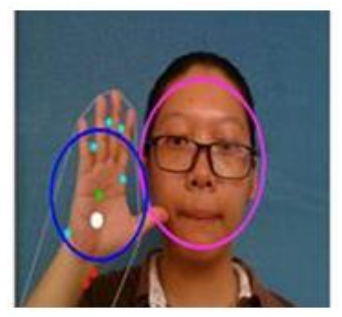

(a)

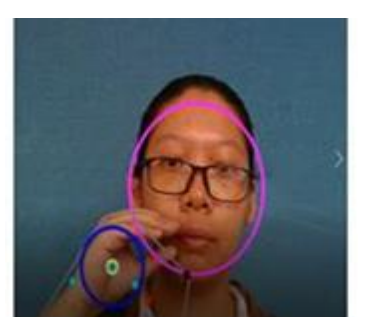

(b)

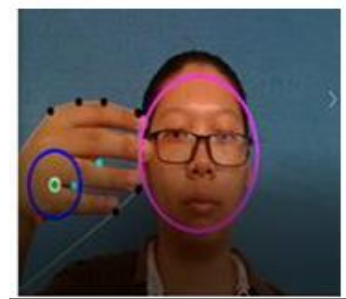

(c)

Figure 3. Recognition of hand gesture (a) mom, (b) eat and (c) milk

\section{RESULTS AND ANALYSIS}

A total of 30 datasets of different races in Malaysia was collected. The datasets were 10 sets for Malay, 10 sets for Chinese and 10 sets for Indian. These 3 main races in Malaysia have been selected to represent 3 different skin colours. 15 datasets were used for training and the remaining 15 datasets were used for testing. Figure 4 shows three signs of baby sign language that are used in this project which are "mom", 
"eat" and "milk". The distance between the laptop and the user is set at $0.3 \mathrm{~m}$. Three experiments were conducted to test the system accuracy in terms of; to investigate the effect of different brightness and different background colour and the overall performance of the developed system based on different skin colour. The accuracy is calculated by using the following formula:

$$
\text { Accuracy }=\frac{\text { Number of test sample that is correctly classified }}{\text { Total test sample }}
$$

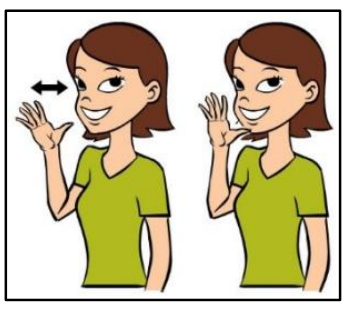

(a)

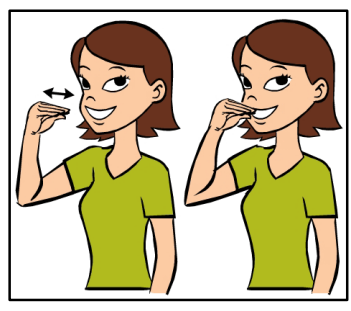

(b)

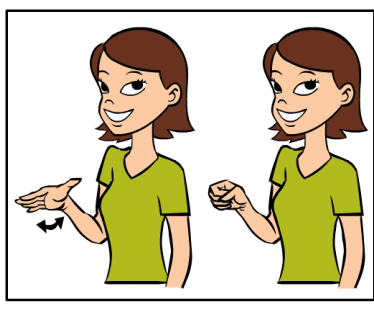

(c)

Figure 4. Three selected signs of baby sign language which are: (a) mom, (b) eat and (c) milk [26]

\subsection{The Effect of Different Brightness}

In brightness test, the accuracy of the system to recognise the hand gesture was tested in common lighting room condition and compared with a setup of external lamp which was placed in front of the laptop. Table 1 shows the result of the hand gesture recognition accuracy in brightness test. It shows that the system is able to recognise the hand gesture more consistent when it is tested with a setup of external lamp that obtained $93.33 \%$ average accuracy than the common lighting with accuracy of only $88.89 \%$. It is because when there is an external lamp placed in front of the user, the hand gesture can be captured more clearly by the system.

Table 1. Hand Gesture Recognition Accuracy in Brightness Test

\begin{tabular}{lcccc}
\hline Brightness condition & \multicolumn{2}{c}{ Hand gesture recognition accuracy (\%) } & Average \\
\cline { 2 - 3 } & Mom & Eat & Milk & accuracy $(\%)$ \\
Common lighting room condition & 100 & 100 & 66.67 & 88.89 \\
External lamp added in front of the laptop & 96.67 & 83.33 & 100 & 93.33 \\
\hline
\end{tabular}

\subsection{The Effect of Different Background Colour}

In background colour test, five types of colour background were selected which included blue, yellow, grey, green and white to determine the best background colour for the system. Table 2 concludes that the system performs the best in blue background which is $94.44 \%$ accuracy whereas the colour performs the worst in yellow background which is $0 \%$ of the accuracy. This huge difference in accuracy is because the yellow colour is actually almost similar to the skin colour tone of human being causing the system to be confused in detecting the face and hand regions. Table 2 shows that green and white backgrounds have the same result of the accuracy which is $92.22 \%$ whereas the grey background shows $90 \%$ accuracy of the gesture recognition. The result proves that the system is able to recognise the hand gesture at different colour background except yellow and the best setup is in blue colour background.

Table 2. Hand Gesture Recognition Accuracy in Background Colour Test

\begin{tabular}{lcccc}
\hline Background & \multicolumn{2}{c}{ Hand gesture recognition accuracy $(\%)$} & Average \\
colour & Mom & Eat & Milk & accuracy $(\%)$ \\
Blue & 100 & 83.33 & 100 & 94.44 \\
Yellow & 0 & 0 & 0 & 0 \\
Grey & 100 & 100 & 70 & 90 \\
Green & 93.33 & 83.33 & 100 & 92.22 \\
White & 93.33 & 83.33 & 100 & 92.22 \\
\hline
\end{tabular}




\subsection{The Overall Performance Comparison on Different Skin Colours}

To evaluate the performance of the system on different skin colours, the background colour was fixed with blue background and with external lamp in front of the laptop. Table 3 shows a summarised result of the effectiveness of the system on different skin color. The result shows that the system can recognise the hand gesture appropriately with different skin colours. Accuracies of $96.33 \%, 85.78 \%$ and $91.78 \%$ of the sign language recognition are yielded for hand gesture "Mom", "Eat" and "Milk" respectively. The lowest accuracy is produced by the sign "eat" followed by the sign "milk". It is due to the difficulty of finding the contour and convexity defects of these sign language if compared to "mom" sign. Furthermore, Malays, Chineses and Indians show 91.33\%, 93.11\% and 89.44\% hand gesture recognition accuracies respectively. Hence, this system is suitable for all races with different skin colours as the implemented skin colour modelling has.

Table 3. Accuracy of Hand Gesture Recognition among Different Races

\begin{tabular}{llccc}
\hline Races & \multicolumn{3}{c}{ Hand gesture recognition accuracy $(\%)$} & Average accuracy \\
\cline { 1 - 3 } Malays & Mom & Eat & Milk & $(\%)$ \\
Chinese & 96.33 & 86.67 & 91 & 91.33 \\
Indian & 96 & 90 & 93.33 & 93.11 \\
& 96.67 & 80.67 & 91 & 89.44 \\
\hline
\end{tabular}

\section{CONCLUSION}

The aim of the present research is to develop a real-time hand gesture recognition system for baby sign language. Two preliminary system setting investigations have been carried out to discover the effect of different light intensity and different background colour. The results have shown that the system has performed the best when an external is added in front of the webcam for the former experiment. Meanwhile, blue colour background has shown a stable average accuracy rate for the latter experiment. From these preliminary setting experiments, they also indirectly expose that the moving background should be avoided to reduce the misclassified hand gesture. However, this study was limited to certain participants since it did not include the children with hearing impairment and babies below 2 years datasets to evaluate the performance of the system towards children. Notwithstanding the limitation sample of children dataset, the system is still applicable to be used as a tool for adult to learn baby sign language. A further study is suggested to test the system by using different age of children with hearing impairment and normal infants below 2 years. In addition, the system can be upgraded into mobile phone apps to make it more portable than the current system and ease the process of image transfer since the image obtained by high end smart phone with great camera is more accurate and clearer than the current one.

\section{ACKNOWLEDGEMENTS}

The work was supported by Universiti Tun Hussein Onn Malaysia (Tier 1 Grant No. H159).

\section{REFERENCES}

[1] "Grades of hearing impairment," World Health Organization, 2019. Retrieved from https://www.who.int/pbd/deafness/hearing_impairment_grades/en/

[2] G. Stevens, et al., "Global and regional hearing impairment prevelance: an analysis of 42 studies in 29 countries," The European Journal of Public Health, vol. 23, pp. 146-152, 2011.

[3] B. O. Olusanya, et al., "The global burden of disabling hearing impairment: a call to action," Bulletin of the World Health Organization, vol. 92, pp. 367-373, 2014.

[4] S. C. Theunissen, et al., "Depression in hearing-impaired children," International Journal of Pediatric Otorhinolaryngology, vol. 75, pp. 1313-1317, 2011.

[5] N. Konuk, et al., "Evaluation of behavioral and emotional problems in deaf children by using the child behavior checklist," Neurology Psychiatry and Brain Research, vol. 13, pp. 59-64, 2006.

[6] T. V. Eldik, et al., "Mental health problems of deaf Dutch children as indicated by parents' responses to the child behavior checklist," American annals of the deaf, vol. 148, pp. 390-395, 2004.

[7] T. V. Eldik, "Mental health problems of Dutch youth with hearing loss as shown on the Youth Self Report," American annals of the deaf, vol. 150, pp. 11-16, 2005.

[8] S. W. Goodwyn, et al., "Impact of symbolic gesturing on early language development," Journal of Nonverbal behavior, vol. 24, pp. 81-103, 2000. 
[9] C. D. Vallotton and C. C. Ayoub, "Symbols Build Communication and Thought: The Role of Gestures and Words in the Development of Engagement Skills and Social-emotional Concepts during Toddlerhood," Social Development, vol. 19, pp. 601-626, 2010.

[10] A. E. Shearer, et al., "Hereditary hearing loss and deafness overview," In GeneReviews ${ }^{\circledR}$ [Internet]. University of Washington, Seattle, 2017.

[11] S. M. Halawani, "Arabic sign language translation system on mobile devices," IJCSNS International Journal of Computer Science and Network Security, vol. 8, pp. 251-256, 2008.

[12] A. Almohimeed, et al., "Arabic text to Arabic sign language translation system for the deaf and hearing-impaired community," In Proceedings of the Second Workshop on Speech and Language Processing for Assistive Technologies, pp. 101-109, 2011.

[13] M. Boulares and M. Jemni, "Mobile sign language translation system for deaf community," In Proceedings of the international cross-disciplinary conference on web accessibility, pp. 37, 2012.

[14] S. Kaur and M. Singh, "Indian Sign Language animation generation system," In 2015 1st International Conference on Next Generation Computing Technologies (NGCT), pp. 909-914, 2015.

[15] M. A. El-Seoud, et al. "Using handheld mobile system for learning sign language," In 15th International Conference on Interactive Collaborative Learning (ICL2012) and 41st International Conference on Engineering Pedagogy, 2010.

[16] J. Wang, et al,. "Chinese sign language animation system on mobile devices," In 2010 Second International Conference on Information Technology and Computer Science, pp. 52-55, July 2010.

[17] I. G. Incertis, et al., "Hand gesture recognition for deaf people interfacing," In 18th International Conference on Pattern Recognition (ICPR'06), vol. 2, pp. 100-103, August 2006.

[18] J. Ravikiran, et al., "Finger detection for sign language recognition," In Proceedings of the international MultiConference of Engineers and Computer Scientists, vol. 1, pp. 18-20, March 2009.

[19] M. S. Abdalla and E. E. Hemayed, "Dynamic hand gesture recognition of arabic sign language using hand motion trajectory features, "Global Journal of Computer Science and Technology, 2013.

[20] E. K. Rishad, et al., "Gesture Controlled Speaking Assistance for Dump and Deaf," IOSR Journal of Computer Engineering (IOSR-JCE), pp. 39-42, 2017.

[21] R. Prakhyath, et al., "Gesture Recognition System," International Journal of Computer Science and Mobile Computing, vol. 7, pp. 164-175, May 2018.

[22] S. S. Rautaray et al., "Vision based hand gesture recognition for human computer interaction: a survey, " Artif. Intell. Rev., vol. 43, no. 1, pp. 1-54, 2012.

[23] G. R. S. Murthy and R.S. Jadon, "A Review of Vision Based Hand Gestures Recognition," International Journal of Information Technology and Knowledge Management, vol. 2, pp. 405-410, 2009.

[24] L. Xingyan, "Gesture Recognition Based on Fuzzy C-Means Clustering Algorithm," Department of Computer Science. The University of Tennessee Knoxville, 2003.

[25] S. Mitra and T. Acharya, "Gesture Recognition: A Survey," IEEE Transactions on systems, Man and Cybernetics, Part C: Applications and reviews, vol. 37, pp. 311-324, 2007.

[26] "Top Ten Starter Signs, "Baby Sign Language.com, 2019. Retrieved from https://www.babysignlanguage.com/dictionary/first-signs/?v=75dfaed2dded

\section{BIOGRAPHIES OF AUTHORS}
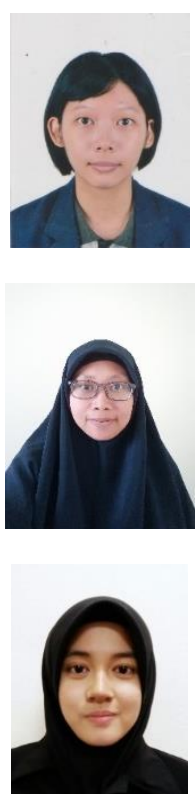

Josephine Ong is a postgraduate student at Universiti Tun Hussein Onn Malaysia (UTHM). She graduated from UTHM with a Bachelor of Electronic Engineering. She is currently involved in a research project to develop a water quality detecting model by using microwave technique. The model should be able to identify the type of contaminants and determine its concentration.

Siti Hajar Aminah Ali is a lecturer at Universiti Tun Hussein Onn Malaysia (UTHM). Her work focuses on image processing and neural network. She received her Bachelor of ElectricalTelecommunications Engineering and Master of Electrical-Electronics and Telecommunications from Universiti Teknologi Malaysia (UTM), Skudai, Malaysia. Her highest education is Doctor of Philosophy in Engineering from Kobe University, Japan.

Ainul Husna Mohd Yusoff is a postgraduate student at Universiti Tun Hussein Onn Malaysia (UTHM). She graduated from Universiti Sains Islam Malaysia (USIM) with Bachelor of Science with Honours (Applied Physics). She pursued her study in Master of Mechanical Engineering at UTHM. Her research project involved the Control Engineering area. 